\title{
A new method for detecting interactions between the senses in event-related potentials
}

Gondan, Matthias; Röder, B.

\section{Published in:}

Brain Research

DOI:

10.1016/j.brainres.2005.12.050

Publication date:

2006

Document version

Publisher's PDF, also known as Version of record

Citation for published version (APA):

Gondan, M., \& Röder, B. (2006). A new method for detecting interactions between the senses in event-related potentials. Brain Research, 1073-1074(1), 389-397. https://doi.org/10.1016/j.brainres.2005.12.050 


\title{
Research Report
}

\section{A new method for detecting interactions between the senses in event-related potentials}

\author{
Matthias Gondan ${ }^{a, *}$, Brigitte Röder ${ }^{b}$ \\ ${ }^{a}$ Department of Experimental Psychology, University of Regensburg, D-93050 Regensburg, Germany \\ ${ }^{b}$ Biological Psychology and Neuropsychology, Hamburg University, Germany
}

\section{A R T I C L E I N F O}

Article history:

Accepted 8 December 2005

Available online 20 January 2006

Keywords:

Divided attention

Reaction time

Event-related potential

Multisensory process

\begin{abstract}
A B S T R A C T
Event-related potentials (ERPs) can be used in multisensory research to determine the point in time when different senses start to interact, for example, the auditory and the visual system. For this purpose, the ERP to bimodal stimuli (AV) is often compared to the sum of the ERPs to auditory $(A)$ and visual $(V)$ stimuli: $A V-(A+V)$. If the result is non-zero, this is interpreted as an indicator for multisensory interactions. Using this method, several studies have demonstrated auditory-visual interactions as early as $50 \mathrm{~ms}$ after stimulus onset. The subtraction requires that $A, V$, and $A V$ do not contain common activity: This activity would be subtracted twice from one ERP and would, therefore, contaminate the result. In the present study, ERPs to unimodal, bimodal, and trimodal auditory, visual, and tactile stimuli (T) were recorded. We demonstrate that $(T+T A V)-(T A+T V)$ is equivalent to $A V-(A+V)$, but common activity is eliminated because two ERPs are subtracted from two others. With this new comparison technique, the first auditory-visual interaction starts around $80 \mathrm{~ms}$ after stimulus onset for the present experimental setting. It is possible to apply the new comparison method to other brain imaging techniques, as well, e.g. functional magnetic resonance imaging.
\end{abstract}

(C) 2005 Elsevier B.V. All rights reserved.

\section{Introduction}

Perception relies heavily on the integration of input from different sensory systems (Welch and Warren, 1986). Basic mechanisms of multisensory integration have been studied with event-related potentials (ERPs). In many of these studies, unimodal and bimodal stimuli were used (e.g. auditory, visual, and auditory-visual stimuli), and the ERP to the bimodal stimulus (AV) was compared to the sum of the ERPs to the unimodal stimuli $(A, V)$ : if the senses operate independently (that is, they form separate 'mental modules'; Sternberg, 2001), the ERP to the bimodal stimulus should be equal to the sum of the ERPs to the unimodal stimuli (Barth et al., 1995): $A V=A+V$, or $A V-(A+V)=0$. By contrast, if the ERP to the bimodal stimulus differs from the sum of the ERPs to unimodal stimuli $(A V \neq A+V)$, it is concluded that the senses interact (Barth et al., 1995). The time point at which the expression $A V-(A+V)$ starts to differ from zero is thought to indicate the processing stage at which the inputs of the different sensory systems are integrated. Using this approach, several studies have demonstrated interactions of the auditory and the visual system (Fort et al., 2002; Giard and Peronnet, 1999; Molholm et al., 2002), the auditory and the somatosensory system (Foxe et al., 2000; Gobbelé et

\footnotetext{
* Corresponding author. Fax: +49941943 3233.

E-mail address: matthias.gondan@psychologie.uni-regensburg.de (M. Gondan).
} 
al., 2003), and the visual and the somatosensory system (Schürmann et al., 2002). In some of these studies, $\mathrm{AV}-(\mathrm{A}+\mathrm{V})$ differed from zero as early as $50 \mathrm{~ms}$ after stimulus onset (e.g. Foxe et al., 2000; Giard and Peronnet, 1999; Molholm et al., 2002), which has been interpreted as evidence for multisensory interactions at early sensory processing stages.

This analysis method has recently been criticized by Teder-Sälejärvi et al. (2002): the authors emphasized that the $\mathrm{AV}-(\mathrm{A}+\mathrm{V})$ subtraction requires that $\mathrm{A}, \mathrm{V}$, and $\mathrm{AV}$ do not elicit any common ERP activity (C). If such common activity exists, it is subtracted twice (AC, VC) from the bimodal ERP (AVC): AVC - $(\mathrm{AC}+\mathrm{VC})=-\mathrm{C}$. Therefore, the resulting term not only reflects interactions of the auditory and visual system, but is also the inverse of this common activity. Two types of common activity can be distinguished: If the auditory and the visual processing pathways converge at jointly used neural structures, this might be considered as a 'real' multisensory interaction. Problems arise if $\mathrm{A}, \mathrm{V}$, and $\mathrm{AV}$ contain unspecific common activity, e.g. activity related to the expectation of the stimulus, or motor preparation. Teder-Sälejärvi et al. demonstrated that the onset of a significant multisensory interaction in ERPs is influenced systematically by the duration of the baseline: Using a baseline correction interval of $-100 \mathrm{~ms}$ to $0 \mathrm{~ms}$ before stimulus onset, AV differed from $A+V$ starting at $60 \mathrm{~ms}$ after stimulus onset. By contrast, a baseline of $-100 \mathrm{~ms}$ to $-50 \mathrm{~ms}$ before stimulus onset moved the first significant auditory-visual interaction to $18 \mathrm{~ms}$ after stimulus onset. The authors suggested that these signs of multisensory interactions did not origin from multisensory processes proper but rather were due to superimposed slow waves such as the contingent negative variation (CNV, Walter et al., 1964). Since the CNV is equally present in $A, V$, and $A V$, it will affect the result of $\mathrm{AV}-(A+V)$ during the entire ERP interval, even before stimulus onset. Teder-Sälejärvi et al. suggested the use of a high-pass filter which eliminates the stimuluspreceding slow deflections in the ERPs to $A, V$, and AV. Indeed, after high-pass filtering, they found a first significant AV - $(A+V)$ interaction at central and parietal electrodes, starting at around $160 \mathrm{~ms}$ after stimulus onset rather than at $50 \mathrm{~ms}$ as reported in earlier studies.

Nevertheless, the stimulus-preceding CNV activity is only one candidate for common activity. For example, it is plausible to assume that processes associated with the P300 (e.g. stimulus evaluation) are active during target detection. These and other processes are part of ' $C$ ', and only a subset of them is eliminated by the high-pass filter. Moreover, the high-pass filter might eliminate low frequency ERP components unique to $A, V$, or $A V$, which are not part of $\mathrm{C}$.

The central aim of the present study is to introduce a new approach to assess auditory-visual interactions in ERPs. In a first step, the ERP to an omitted stimulus ( $O$, 'nullstimulus') is added to the minuend side of the comparison: $(\mathrm{O}+\mathrm{AV})-(\mathrm{A}+\mathrm{V})$. If the omitted stimulus elicited only $\mathrm{C}$, it would be eliminated because two ERPs are subtracted from two others. Unfortunately, omitted stimuli may elicit rather specific ERP deflections (a prolonged CNV, and the so-called 'missing stimulus related potential' Busse and Woldorff, 2003; Simson et al., 1976). Therefore, in a second step, each stimulus is presented together with an additional tactile stimulus $(T)$.

The ERP comparison is now $(T+T A V)-(T A+T V)$. Unisensory ERP activity and common activity are eliminated in this comparison. Theoretically, the trimodal stimulus elicits additional ERP activity due to the interaction of the auditory and the visual system, the auditory and the tactile system, the visual and the tactile system, and possibly even trisensory interactions (Table 1). However, auditory-tactile and visuotactile interactions should be eliminated because both should be equally visible in TA and TV. Therefore, auditory-visual and-if present-trisensory interactions are isolated in the comparison.

An ERP study was run to compare the two methods. ERPs to uni-, bi-, and trimodal auditory, visual, and tactile stimuli were recorded, while participants had to make speeded responses to infrequent target stimuli. Multisensory interactions were investigated using the new comparison $(T+T A V)-(T A+T V)$. The results were compared with those of the 'classical' analysis approach, AV - $(A+V)$. A

\begin{tabular}{|c|c|c|c|c|c|c|c|c|c|}
\hline Method & Stimuli & Common & SEP & AEP & VEP & $A \times V$ & $A \times T$ & $\mathrm{~V} \times \mathrm{T}$ & $A \times V \times T$ \\
\hline \multicolumn{10}{|c|}{$(T+T A V)-(T A+T V)$} \\
\hline & $+\mathrm{T}$ & + & + & & & & & & \\
\hline & $+\mathrm{TAV}$ & + & + & + & + & + & + & + & $(+)$ \\
\hline & $-\mathrm{TA}$ & - & - & - & & & - & & \\
\hline & $-\mathrm{TV}$ & - & - & & - & & & - & \\
\hline & Result & Zero & Zero & Zero & Zero & $A \times V$ & Zero & Zero & $(A \times V \times T)$ \\
\hline \multicolumn{10}{|c|}{$A V-(A+V)$} \\
\hline & $+\mathrm{AV}$ & + & & + & + & + & & & \\
\hline & $-A$ & - & & - & & & & & \\
\hline & $-\mathrm{V}$ & - & & & - & & & & \\
\hline & Result & -Common & & Zero & Zero & $A \times V$ & & & \\
\hline
\end{tabular}

Common: common ERP activity, for example, the contingent negative variation or the P300-SEP, AEP, VEP: somatosensory, auditory, and visual evoked potential- $\mathrm{A} \times \mathrm{V}, \mathrm{A} \times \mathrm{T}, \mathrm{V} \times \mathrm{T}, \mathrm{A} \times \mathrm{V} \times \mathrm{T}$ : auditory-visual, auditory-tactile, visuo-tactile, and trisensory interactions. 
new variant of the race model test was developed to check for redundancy gains due to trisensory interactions (Gondan and Röder, in revision; for the general procedure, see Miller, 1982).

\section{Results}

\subsection{Reaction time data}

False alarms and misses were below $10 \%$ on average (i.e., less than 4 misses per target condition) and were not further analyzed. Reaction times for the seven types of target stimuli are shown in Table 2: responses to trimodal targets were fastest followed by responses to bimodal targets, and responses to unimodal targets were slowest (unimodal vs. bimodal: $t_{18}=12.3, P<0.01$; bimodal vs. trimodal: $\left.t_{18}=7.9, P<0.01\right)$. The reaction time gain in bimodal redundant targets (AV, TA, TV) was larger than predicted by the race model (Eq. (1), Fig. 1a shows AV). Whereas the race model test of Eq. (4) indicated coactivation (Fig. 1B), a specific trisensory coactivation effect was not observed (Eq. (5), Fig. 2B). The reaction times thus indicate that the simultaneous presentation of the auditory and visual stimuli resulted in coactivation (Figs. 1a, b, 2b) but that the redundancy gains due to bisensory coactivation were sufficient to explain the redundancy gain in responses to trimodal stimuli. Significant violations of the race model are indicated in the figures.

\subsection{ERP data}

Event-related potentials (ERPs) to auditory, visual, and tactile stimuli are depicted in Fig. 3. The auditory ERP showed characteristic deflections at $\mathrm{Cz}$ (Pa: $40 \mathrm{~ms}, \mathrm{P} 1: 70 \mathrm{~ms}, \mathrm{~N} 1: 110$ ms, P2: $200 \mathrm{~ms}, \mathrm{N2}: 300 \mathrm{~ms}$, and a small P3 around $350 \mathrm{~ms}$ ). The visual ERP was characterized by a P1-N1 deflection at occipital recording sites, around $120 \mathrm{~ms}$ and $170 \mathrm{~ms}$, respectively. The tactile stimuli elicited a somatosensory ERP over central recording sites of the left hemisphere, contralateral to the stimulated hand. The ERP to the omitted stimulus consisted of a slow positive ramp-like wave over the whole scalp.

Evidences for multisensory interactions of the auditory and the visual system, as revealed by the result of the ERP difference $(T+T A V)-(T A+T V)$, are displayed in Fig. 4 (solid lines). An initial interaction was visible as a

Table 2 - Mean reaction times in milliseconds to target stimuli

\begin{tabular}{lll} 
Stimulus & RT & SE \\
\hline A & 511 & 21 \\
V & 530 & 21 \\
T & 544 & 24 \\
AV & 461 & 19 \\
AT & 482 & 19 \\
VT & 483 & 18 \\
AVT & 449 & 21 \\
\hline SE: standard error. & & \\
\hline
\end{tabular}
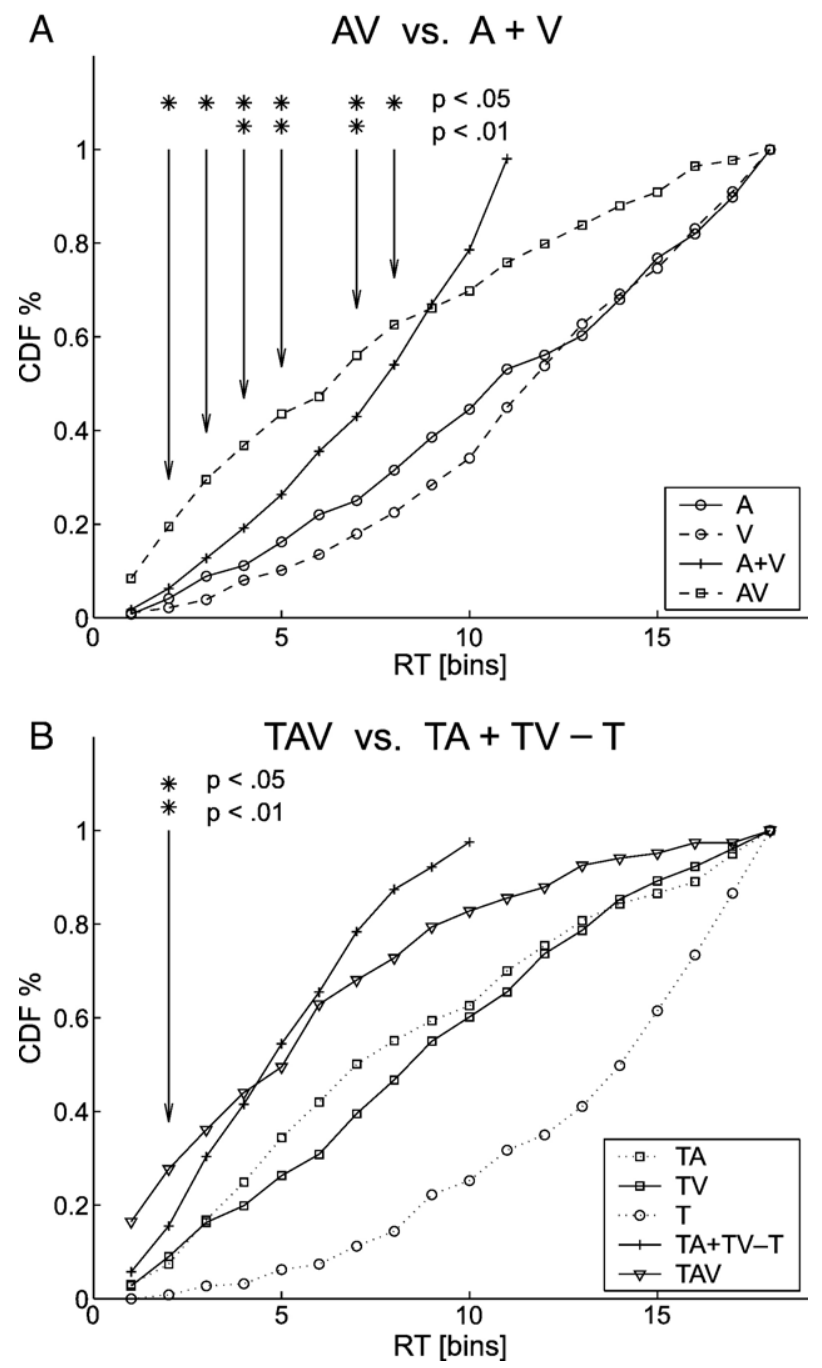

Fig. 1 - Race model test for auditory-visual stimuli: The left figure (A) shows the results of the race model test described by Miller (1982) for auditory, visual, and bimodal auditory-visual stimuli (Eq. (1)): $p(\mathrm{AV}) \leq p(\mathrm{~A})+p(\mathrm{~V})$. The right figure shows the modified race model test by Diederich (1992), in which the CDF of the trimodal stimulus is contrasted to the CDF of the summed auditory-tactile and visuo-tactile stimuli, minus the $\mathrm{CDF}$ of the reaction times to unimodal tactile stimuli (Eq. (4)): $p(\mathrm{TAV}) \leq p(\mathrm{TA})+p(\mathrm{TV})-p(\mathrm{~T})$. In panels $A$, the same stimuli are used as in the 'classical' ERP comparison $A V-(A+V)$, whereas in panels $B$, the stimuli of the new ERP comparison are used (T + TAV) - TA + TV). In both tests, the reaction time gain outgrows the race model prediction; hence, auditory-visual coactivation can be concluded.

negative deflection over T8 and emerged starting at 84 ms after stimulus onset. A further interaction was observed over frontal and temporo-parietal regions, between $140 \mathrm{~ms}$ and $180 \mathrm{~ms}$ after stimulus onset. Its maximum was located over the right temporal lobe. An additional interaction started around $250 \mathrm{~ms}$ after stimulus onset and emerged as a relative negativity over central recording sites. 

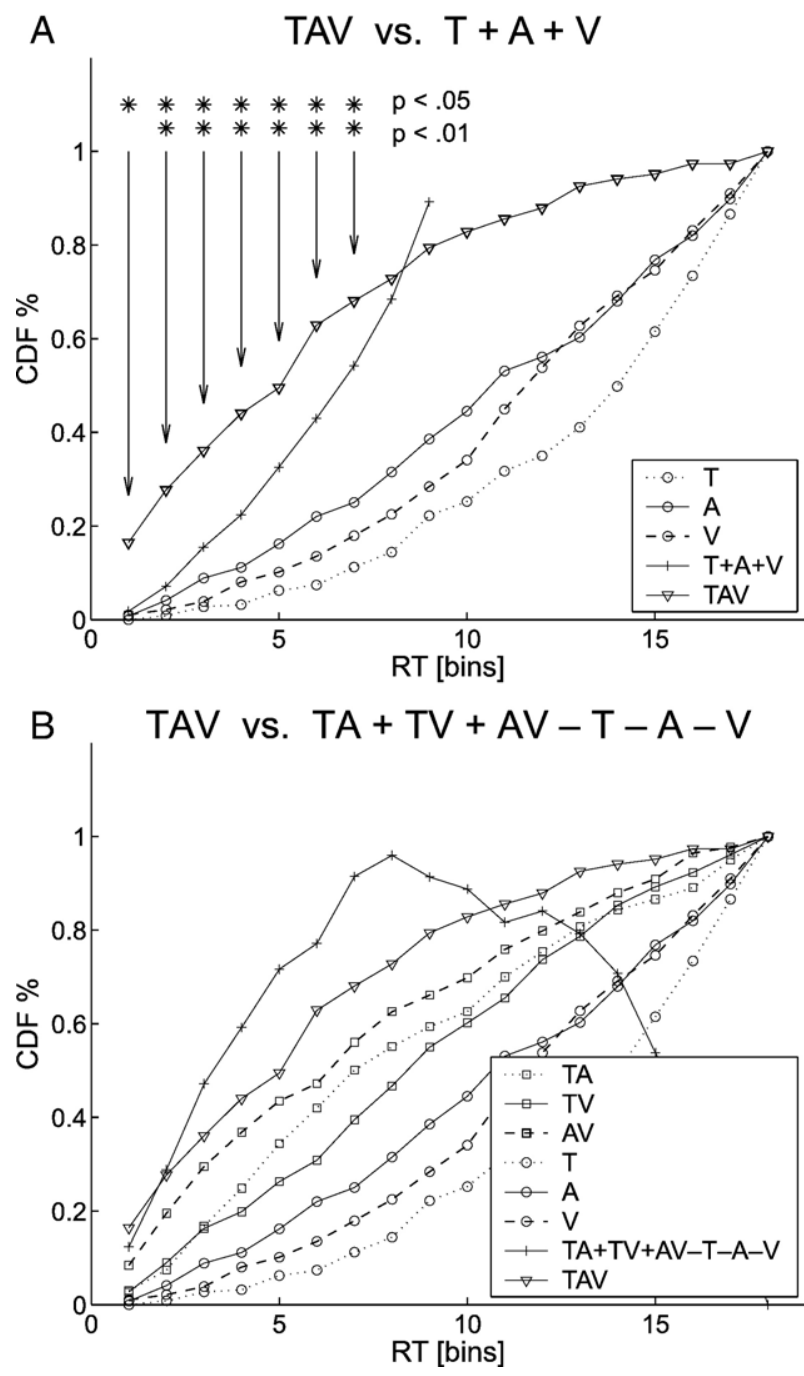

Fig. 2 - Trimodal race model test for TAV stimuli. The left figure compares the reaction time CDF of TAV stimuli to the reaction time CDFs of $T, A$, and $V$ stimuli (Eq. (2)). It indicates coactivation at a large percentile range, but the origin of this coactivation is equivocal (see text). The right test checks for trisensory coactivation (Eq. (5)): $p(\mathrm{TAV})+p(\mathrm{~T})+p(\mathrm{~A})+p(\mathrm{~V}) \leq$ $p(\mathrm{AV})+p(\mathrm{TA})+p(\mathrm{TV})$. It turns out that the redundancy gain in the responses to trimodal stimuli can entirely be explained by bisensory coactivation.

During the first $200 \mathrm{~ms}$ after stimulus onset, the results of the ERP difference AV - $(A+V)$ were very similar to the results revealed by the new method (Fig. 4, dotted lines). However, starting at $350 \mathrm{~ms}, \mathrm{AV}-(\mathrm{A}+\mathrm{V})$ indicated a negative deflection over centroparietal leads, which was not observed in $(\mathrm{T}+\mathrm{TAV})-(\mathrm{TA}+\mathrm{TV})$.

The differences between the ERP comparison using the "old" method $[\mathrm{AV}-(\mathrm{A}+\mathrm{V})]$ and the ERP comparison with the "new" method $[(T+T A V)-(T A+T V)]$ as well as the ERP to the omitted stimulus are shown in Fig. 5. During the first $200 \mathrm{~ms}$ after stimulus onset, "new-old" is similar to the ERP to the omitted stimulus. At later stages, "newold" shows a P3-like deflection. This deflection is not observed in the omitted stimulus ERP.

\section{Discussion}

The present study introduces a new procedure for investigating multisensory interactions with event-related potentials (ERPs). We demonstrated weaknesses of the previously used analysis method in which the bimodal evoked potential was compared to the sum of the unimodal evoked potentials. A different approach to deal with these problems by using stimuli of a third modality was outlined. The new ERP measure $(T+T A V)-(T A+T V)$ is formally equivalent to $A V-(A+V)$, that is, auditory-visual interactions are isolated (see Table 1, but see discussion of trisensory interactions below). Since two ERPs are subtracted from two others, ERP activity common to the different stimulus conditions is eliminated. Using this approach, we demonstrate a first auditory-visual interaction starting at $84 \mathrm{~ms}$ at central recording sites. This finding is in accordance with Teder-Sälejärvi et al. (2002, Fig. 6, p. 113) who, however, attributed this early deflection to residual portions of a stimulus-preceding CNV which were not eliminated by the high-pass filter employed in their study. Similarly as TederSälejärvi et al., the present study did not find multisensory interactions as early as $50 \mathrm{~ms}$ after stimulus onset (e.g. Giard and Peronnet, 1999).

The positivity around $150 \mathrm{~ms}$ (temporo-parietal distribution) and the negativity around $250 \mathrm{~ms}$ (central topography) were also reported by Teder-Sälejärvi et al. (2002). In addition to 'conventional' ERP analysis, Teder-Sälejärvi et al. used dipole modeling to localize the sources of the observed interactions. Giard and Peronnet (1999) suggested that the interaction at $150 \mathrm{~ms}$ might reflect a decrease of the visually evoked N1, that is, the auditory stimulus modulates the activity of higher-order visual areas. Indeed, the dipole analysis indicated a source in the inferior parieto-occipital region (Teder-Sälejärvi et al., 2002). The source of the third interaction was localized in the anterior temporal association cortex and in polymodal areas of the superior temporal cortex.

In all three modality pairings, statistical facilitation could not account for the reaction time gained by bimodal stimulation. Therefore, a coactivation model was accepted, although it is difficult to derive the processing stage at which the information of the sensory systems is integrated from the present data. Further studies are needed to shed light on the nature of the redundant target effect in bimodal divided attention, for example, by using different modality pairings or varying the spatial or temporal relationship of the stimulus components (Gondan et al., 2005; Murray et al., 2005; Schürmann et al., 2002).

Trimodal stimuli, as used on the left side of the ERP comparison (TAV), might not only have elicited auditory, visual, tactile, and auditory-visual ERPs, but also specific auditorytactile, visuo-tactile, and possibly trisensory ERP interactions which might contaminate the result of the new comparison. We tried to minimize interactions of the tactile modality with the auditory and/or visual modality by using tactile stimuli which were spatially separated from the two other sources and which were presented out of the focus of attention. It has been shown that bisensory interactions observed for neurons of the superior colliculus heavily depend on the spatial relationship of two stimuli (Wallace et al., 1992), although, at the cortical level, bisensory interactions might not always follow this spatial 

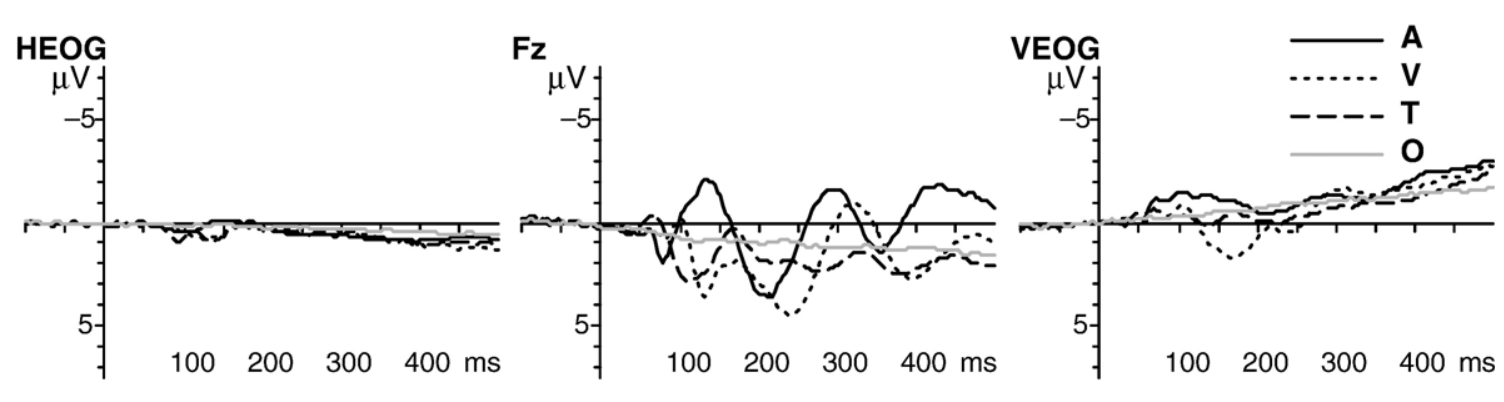

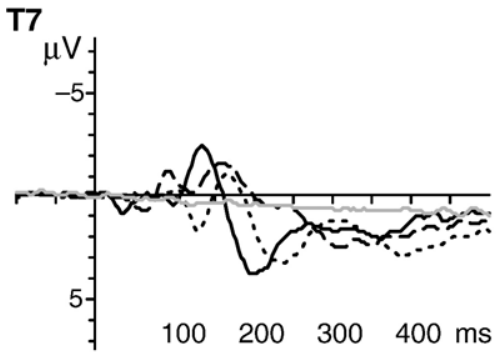

$\mathrm{Cz}$

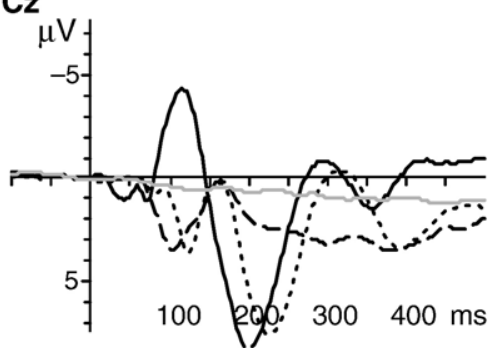

P5

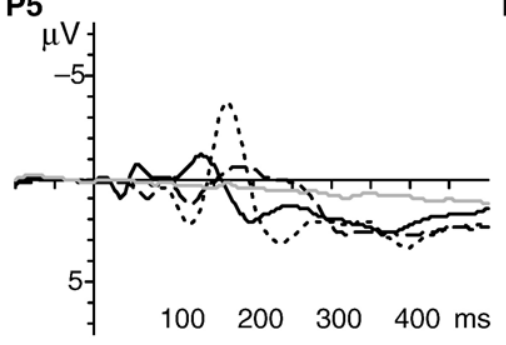

$\mathrm{POz}$

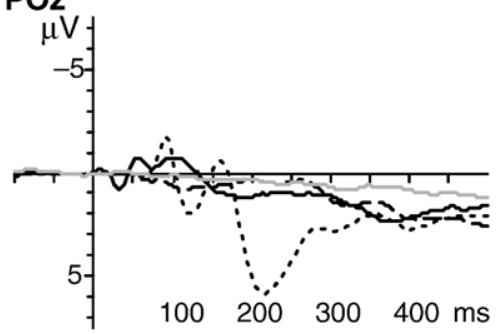

T8

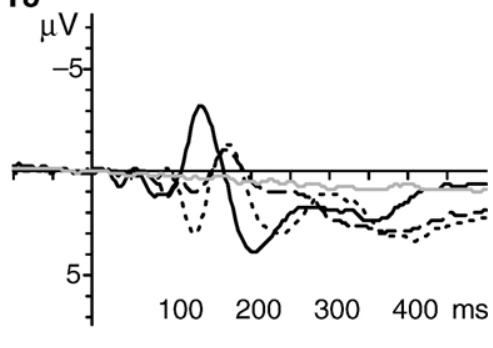

P6

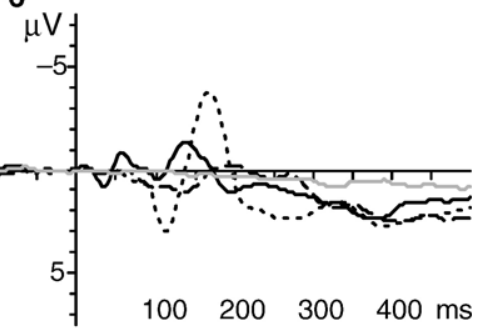

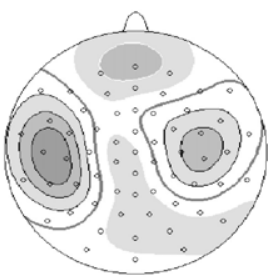

$\mathrm{T}: 150 \ldots 170 \mathrm{~ms}$

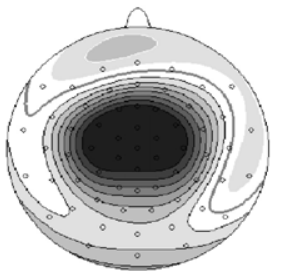

$\mathrm{A}: 100 \ldots 120 \mathrm{~ms}$

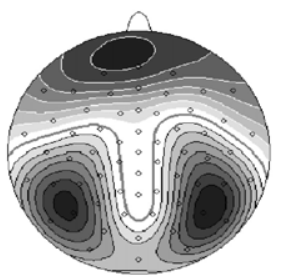

$\mathrm{V}: 150 \ldots 170 \mathrm{~ms}$

Fig. 3 - Event-related potentials to auditory (A), visual (V), tactile (T), and 'omitted' stimuli (O).

congruity rule (e.g. Murray et al., 2005). In any case, auditorytactile and visuo-tactile interactions should be eliminated because they are elicited by the stimuli on the right side of the expression (TA, TV), as well (Table 1). However, trisensory interactions, i.e., neural responses unique to trimodal stimuli which cannot be explained by interactions of the auditoryvisual, the auditory-tactile, or the visual-tactile system, remain in the expression and might contaminate the results. Despite some reports of trimodal cells in the superior colliculus (Wallace and Stein, 2001) and in the parietal cortex (auditory-visualvestibular: Schlack et al., 2005), trisensory interactions, i.e., specific responses to trimodal events, have not been investigated systematically yet, neither behaviorally nor on the neural level. Given that a trimodal stimulus is already highly 'amplified' due to auditory-visual, auditory-tactile, and visuo-tactile integration mechanisms, a system which exclusively integrates trimodal events seems of little use. This is confirmed by the reaction time analysis of the present study: shorter reaction times to trimodal stimuli could be entirely explained by bisensory coactivation (Eq. (5) and Fig. 2b). It should be noted, however, that the problem of trisensory interactions is far from settled. Given the enormous complexity of the calculations needed to map the different spatial representations onto each other (vision: retina, audition: head, touch: body surface), it might be well possible that the brain integrates the information of the different modalities in a pairwise manner.

We argued that ERP activity C common to A, V, and AV was the problem of the AV - $(A+V)$ comparison. Since this activity $C$ is subtracted twice from one ERP, $A V-(A+V)$ is reflecting the multisensory interaction of audition and vision (MSI), minus the common activity: AVC - (AC + VC) = MSI - C. In the comparison suggested in the present study, this common activity is eliminated because two ERPs are subtracted from two others: $($ TC + TAVC $)-($ TAC + TVC $)=$ MSI. We can, therefore, estimate C by subtracting the results obtained by the old method from the results obtained by the new method: MSI $-($ MSI $-C)=$ C. Fig. 5 shows that $C$ is very similar to the event-related potential to the omitted stimulus (O) until $200 \mathrm{~ms}$ after stimulus onset. Both C 


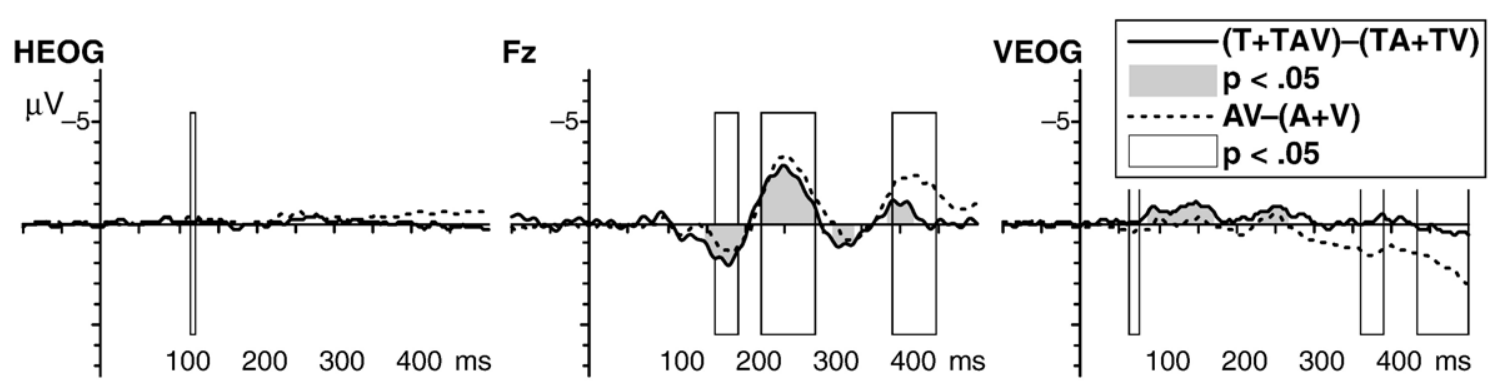

T7

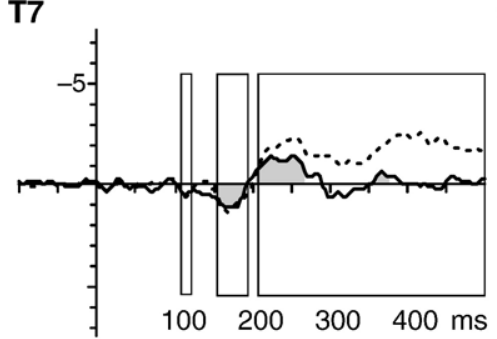

$\mathrm{Cz}$

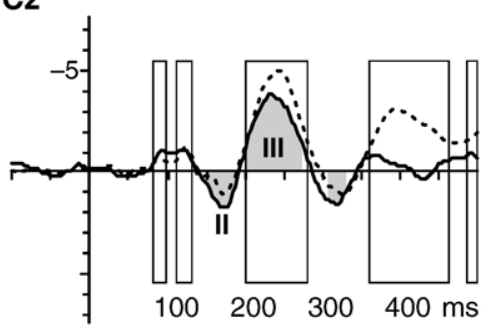

T8

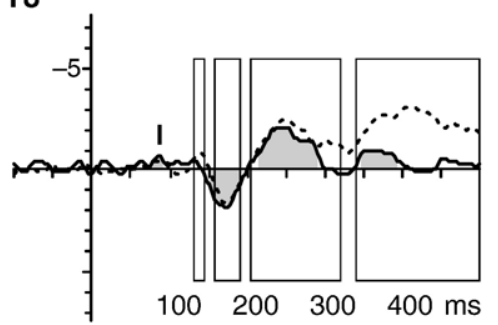

P5

$\mathrm{POz}$
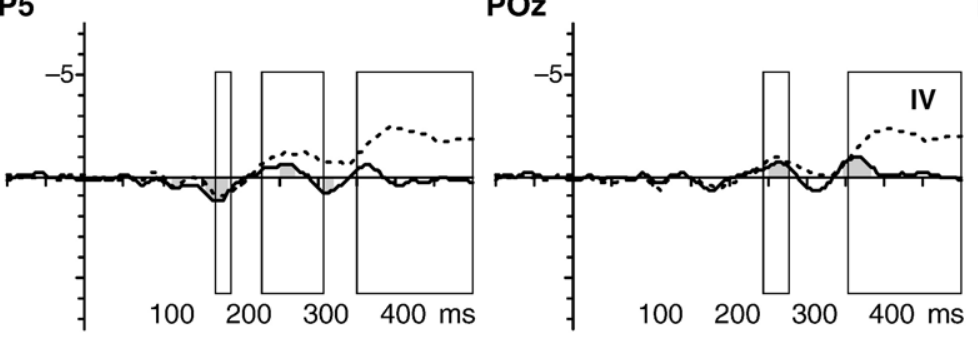

P6
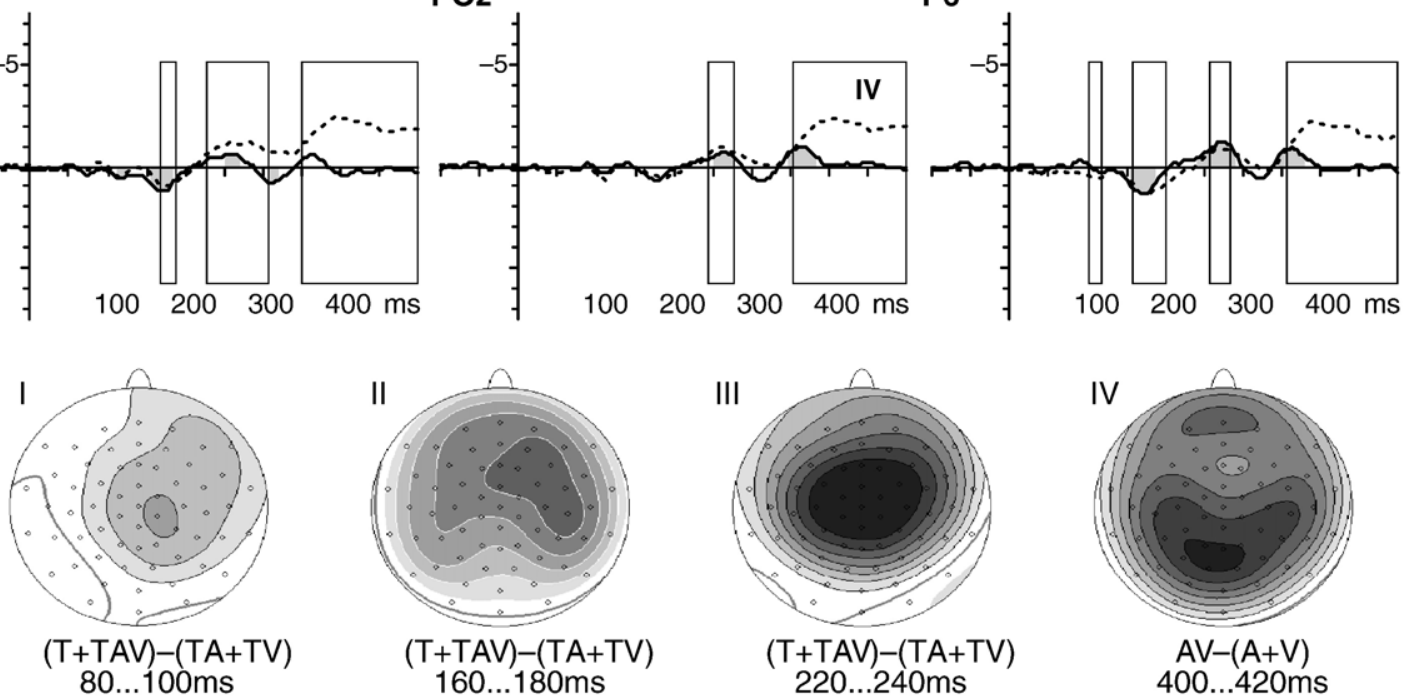

Fig. 4 - Multisensory interactions as indicated by $(T+T A V)-(T A+T V)$ are shown in solid lines, the respective results of AV $-(A+V)$ are shown in dotted lines. The shades and rectangles indicate where the expressions differ significantly from zero for at least $10 \mathrm{~ms}(P<0.05)$. Until $200 \mathrm{~ms}$, the two methods yield similar results: a weak first interaction is peaking around $90 \mathrm{~ms}$ after stimulus onset (new method: significant at T8, old method: significant at Cz). A second interaction emerges around $160 \mathrm{~ms}$, with a broad bilateral fronto-temporal positivity followed by a third interaction around $250 \mathrm{~ms}$, visible as a centrally localized negativity. At later processing stages, $A V-(A+V)$ indicates a parietally localized negative deflection, possibly due to the P3 wave which is subtracted twice from the ERP to the AV stimulus.

and $O$ elicit a ramp-like deflection which might reflect that the participant is preparing for the next stimulus. In consequence, it could be argued that, in simple tasks like the one reported in the present study, adding the omitted stimulus response to the left side of the ERP comparison should take care of anticipatory potentials: $(O+A V)-(A+V)$. This is especially due to the observation that the omitted stimulus response was weak, possibly due to the irregular inter-stimulus interval and due to the fact that-in contrast to Simson et al. (1976)-the omitted stimuli did not have any response relevance. However, in the later time course, $\mathrm{C}$ differs from $\mathrm{O}$. This is illustrated by the P3like deflection in Fig. 5 which is elicited only by the real stimuli and is, therefore, part of $\mathrm{C}$.
It has to be noted that $(T+T A V)-(T A+T V)$ does not eliminate each activity common to A, V, and AV: if a neural structure is activated in the same way by auditory, visual, and auditory-visual stimuli (e.g. with an activity level of 1 unit), both methods should formally yield the same result: $\mathrm{AV}-(\mathrm{A}+\mathrm{V})=1-(1+1)=-1$ and $(T+\mathrm{TAV})-(\mathrm{TA}+\mathrm{TV})=(0+1)-(1+1)=-1$. Therefore, the new method is able to distinguish such sub-additive interactions $(A V<A+V)$ in the same way as $A V-(A+V)$.

In a somewhat similar approach as the one introduced in the present study, Schröger and Widmann (1998) applied the $\mathrm{AV}-(\mathrm{A}+\mathrm{V})$ logic to an oddball paradigm with four types of auditory-visual stimuli (AV: both standard, A*V: auditory 


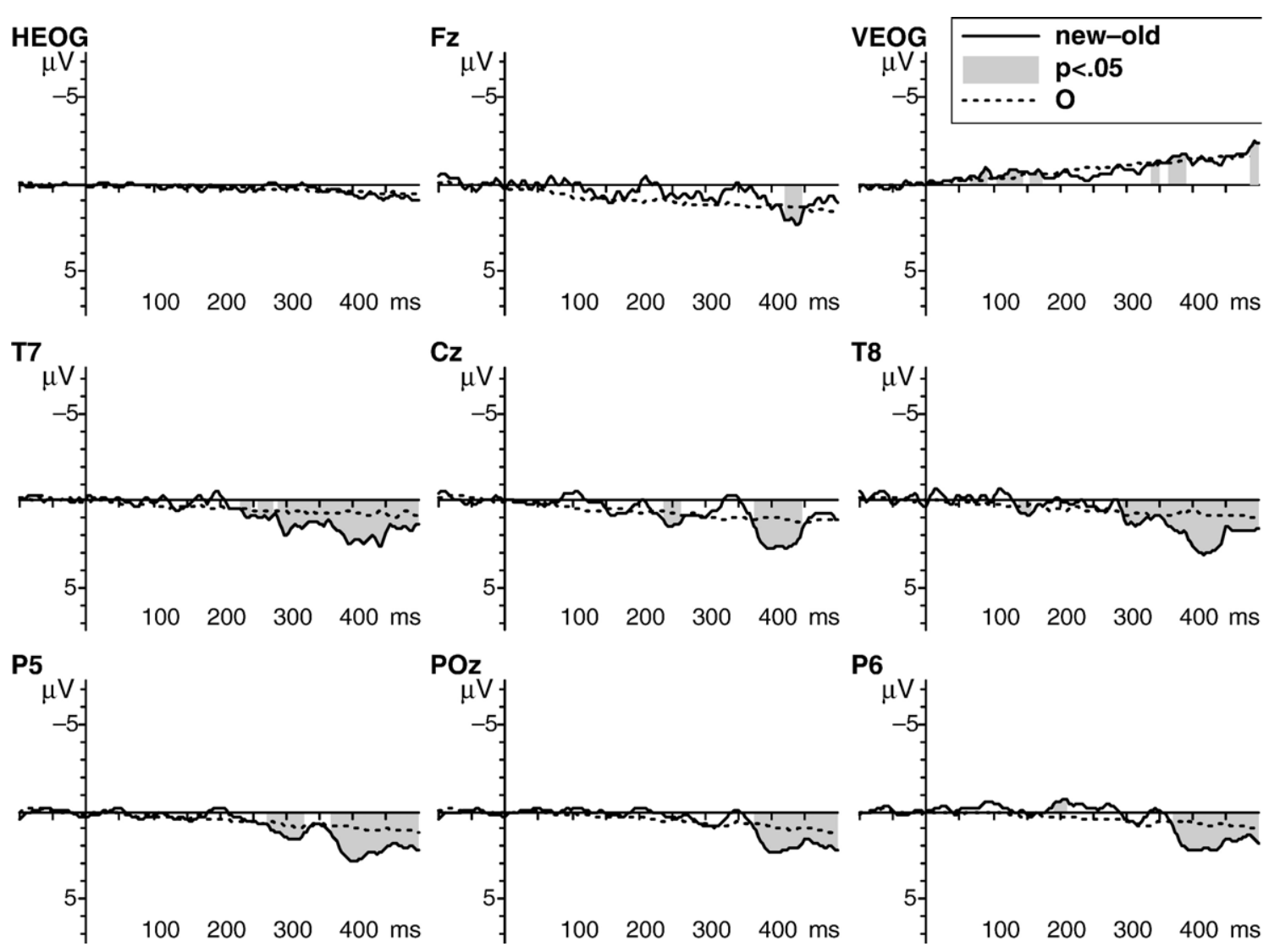

Fig. 5 - The difference ("new minus old") between (T + TAV) - (TA + TV) and AV - (A + V) is shown in solid lines. The shades indicate the intervals in which the results of the two methods differ significantly $(P<0.05$, at least $10 \mathrm{~ms})$. The ERP to the omitted stimulus is shown in dotted lines. "new-old" and the omitted stimulus yield similar ramp-like deflections in the first 300 ms. Later on, the two curves differ, presumably due to the P3 artifact observed only in "new-old".

deviant and visual standard, $\mathrm{AV}^{*}$ : auditory standard/visual deviant, $\mathrm{A}^{*} \mathrm{~V}^{*}$ : both deviants). They tested if the effects of the auditory and the visual deviant add linearly by evaluating $\left(A^{*} V^{*}-A V\right)-\left[\left(A^{*} V-A V\right)+\left(A V^{*}-A V\right)\right]$. This term can be rewritten as $\left(A V+A^{*} V^{*}\right)-\left(A^{*} V+A V^{*}\right)$. It is evident that, with this symmetric comparison, early unspecific common activity (e.g. the CNV) is eliminated. At later stages, the modalityunspecific components of the ERPs might be different, for example, only the deviants $\left(A^{*} V^{*}, A^{*} V, A V^{*}\right)$ elicit a P300 deflection. The resulting non-additivity would indicate that the two sensory channels converge to common neural representations and that the processing of, e.g. an auditory target, is not independent of the presence or absence of a visual target. In this respect, non-additivity at later components can be taken as evidence for a supramodal process.

Since the latency of later non-sensory components (e.g. the P300) might be different in the ERPs to T, TAV, TA and $\mathrm{TV}$, the expression $(\mathrm{T}+\mathrm{TAV})-(\mathrm{TA}+\mathrm{TV})$ can be non-zero at later stages, even though these ERPs might have similar waveforms (Sheila G. Crewther, La Trobe University, Victoria, Australia, personal communication, IMRF 2004 meeting). Formally seen, a latency change due to multisensory stimulation is nothing else than a multisensory interaction. Nonetheless, the topography of the ERP difference at later processing stages should be interpreted with caution. This applies for both $(T+T A V)-(T A+T V)$ and $A V-(A+V)$.
Although the present study focused on auditory-visual interactions measured by event-related potentials, the introduced approach is neither limited to ERPs nor to auditoryvisual interactions: it can as easily be extended to magnetoencephalography and to functional magnetic resonance imaging studies. Interactions of audition and touch can be investigated using the expression $(\mathrm{V}+\mathrm{VAT})-(\mathrm{VA}+\mathrm{VT})$, interactions of vision and touch can be investigated using the expression $(A+A V T)-(A V+A T)$. Most important, since the new method is robust against common activity, it is possible to investigate multisensory interactions for larger time intervals and in more complex tasks than with the classical $\mathrm{AV}-(\mathrm{A}+\mathrm{V})$ method.

\section{Experimental procedure}

\subsection{Participants}

Nineteen students (3 left-handed, 14 female, age between 19 and 23 years) participated. All were free of neurological disorders and had normal hearing and normal or corrected-to-normal vision. They received course credits or monetary compensation for participating and gave written informed consent. EEG data from three other participants had to be discarded due to a low signal-tonoise ratio in the ERP data. 


\subsection{Stimuli and task}

The experiment used an oddball paradigm with $90 \%$ 'standard' stimuli which did not require any response, and 10\% 'target' stimuli to which participants had to respond. Unimodal auditory, visual and tactile stimuli, bimodal auditory-visual, auditory-tactile, and visuo-tactile stimuli, and trimodal stimuli were presented either as single stimuli (20 ms, standards) or twice (20 ms stimulus, $100 \mathrm{~ms}$ gap, $20 \mathrm{~ms}$ stimulus, targets), in random order. The auditory stimuli were short noise bursts (67 $\mathrm{dB})$, delivered by a loudspeaker in front of the participant, at a distance of $1 \mathrm{~m}$. The visual stimuli were delivered with two red LEDs (60 mcd) which were mounted directly onto the top of the loudspeaker. Tactile stimuli consisted of metallic posts (diameter $0.5 \mathrm{~mm}$ ) which were moved up by $0.5 \mathrm{~mm}$ to touch the right index finger. To mask the faint noise generated by the tactile stimulator, it was placed into a sound-absorbing box. The participants were instructed to respond as fast as possible to any target by releasing a foot pedal with the right foot. Each standard was presented 405 times, and each target was presented 45 times during the experiment. In addition, 405 gaps (omitted stimuli) were mixed into the stimulus sequence, in which no stimulus was presented, but a trigger signal was sent to EEG recording system. The inter-stimulus interval varied randomly between $1300 \mathrm{~ms}$ and $1700 \mathrm{~ms}$. The entire session was divided into 15 blocks of about $5 \mathrm{~min}$ of duration each. Altogether, the experiment took about $2 \mathrm{~h}$. Between the blocks, participants received feedback on misses and false alarms.

\subsection{EEG recording}

The EEG was recorded from 61 equidistantly located scalp electrodes (non-polarizable $\mathrm{Ag} / \mathrm{AgCl}$ electrodes) mounted into an elastic cap (Easy Cap, FMS). The left mastoid served as the reference. The electrode impedance was kept at $5 \mathrm{k} \Omega$ or below by preparing the skin with 'Every' (gelimed) and isopropyl alcohol. Eci Electrogel (Electrocap International, Inc.) was used as electrolyte for all recordings. The band pass of the amplifiers (Neuroscan) was set from $0.1 \mathrm{~Hz}$ to $100 \mathrm{~Hz}$; the sampling rate was $500 \mathrm{~Hz}$. Horizontal eye movements were monitored with a bipolar recording of two electrodes attached to the outer canthi of the eyes. Vertical eye movements were measured with an electrode placed under the left eye, against the reference.

\subsection{ERP data analyses}

Only ERPs to standards were analyzed. The ocular channels served for off-line rejection of trials with eye blinks and excessive eye movements. Segments with ocular activity larger than $50 \mu \mathrm{V}$ within the epoch between $100 \mathrm{~ms}$ before and $500 \mathrm{~ms}$ after stimulus onset were rejected. ERPs to each condition were averaged separately, baseline-corrected to the mean activity between 50 $\mathrm{ms}$ and $0 \mathrm{~ms}$ before stimulus onset, and re-referenced off-line to the mean voltage at both mastoids.

To investigate multisensory interactions, the ERP elicited by the single tactile stimulus and the ERP of the trimodal standard were summed and compared to the summed ERP activity to the auditory-tactile and visuo-tactile stimuli: $(T+T A V)$ - (TA + TV). The physical stimulation in this difference is zero because the unimodal stimulus components are identical for minuend and the subtrahend. As shown in Table 1, the same should hold for auditory-tactile and visuo-tactile interactions. In contrast, ERP activity elicited by the interaction of the auditory and the visual system is unique to TAV and should, therefore, be isolated in the result. A testing procedure for trisensory redundancy gains in the reaction times to trimodal stimuli will be introduced in the next section.

Epochs during which ( $\mathrm{T}+\mathrm{TAV})-(\mathrm{TA}+\mathrm{TV})$ differed from zero at a specific electrode were considered reliable if this difference remained significant $(P<0.05)$ for more than five consecutive sampling points (10 ms). The results were compared with the respective findings of $A V-(A+V)$.

\subsection{Reaction time analysis}

Mean reaction times (RTs) for auditory-visual target stimuli are typically below the mean RTs for unimodal auditory or visual stimuli. Two models have been suggested to explain this finding (redundant-signals effect): the race model and the coactivation model (Miller, 1982). The race model assumes that the information of the two sensory channels is processed independently and that the channel which has finished processing fastest triggers the response. Consequently, the probability to produce a fast response is higher for bimodal stimuli than for unimodal stimuli, like the probability to obtain a six is higher when two dice are tossed instead of one. The reaction time gain obtained by this mechanism (statistical facilitation, Raab, 1962) has an upper limit, which is described by the race model inequality (Miller, 1982):

$p\left(\mathrm{RT}<\mathrm{t}_{0} \mid \mathrm{AV}\right) \leq p\left(\mathrm{RT}<\mathrm{t}_{0} \mid \mathrm{A}\right)+p\left(\mathrm{RT}<\mathrm{t}_{0} \mid \mathrm{V}\right)$

short: $p(\mathrm{AV}) \leq p(\mathrm{~A})+p(\mathrm{~V})$

For example, if $10 \%$ of the responses to auditory stimuli and $5 \%$ of the responses to visual stimuli are faster than $t_{0}=200 \mathrm{~ms}$, the race model predicts that the proportion of responses to bimodal stimuli which are faster than $200 \mathrm{~ms}$ is below or equal to $15 \%$, which is the sum of the two proportions. If this inequality is violated, a coactivation model is adopted, which assumes that the origin of redundancy gain in bimodal stimuli is due to multisensory integration at a certain processing stage.

The race model test can easily be extended to trimodal stimuli (Diederich, 1992):

$p(\mathrm{TAV}) \leq p(\mathrm{~T})+p(\mathrm{~A})+p(\mathrm{~V})$

If Eq. (2) is violated, though, the origin of the redundancy gain remains equivocal: theoretically, TAV may elicit redundancy gains due to the simultaneously presented auditory, visual, and tactile stimulus components, but also due to the auditory-visual, auditory-tactile, or visuo-tactile stimulus implicitly included in TAV. In other words, bisensory and trisensory interactions may result in a reaction time gain and increase $p(\mathrm{TAV})$.

To isolate the redundancy gain due to trisensory interactions, the bisensory coactivation effects have to be included on 
the right side of the comparison (Gondan and Röder, in revision):

$p(\mathrm{TAV}) \leq p(\mathrm{TA})+p(\mathrm{~V})$

In a first step, $p(T)+p(A)$ is replaced by $p(T A)$. TAV is thus considered to be a combination of the bimodal stimulus TA and the unimodal stimulus V. If Eq. (3) turns out to be invalid, it is unlikely that the redundancy gain would be due to auditory-tactile coactivation because the latter would equally increase both sides of the inequality. However, the exact locus of coactivation would still be unspecified since three candidates remain, namely, the auditory-visual, visuo-tactile, and trisensory interactions.

In a second step, $p(T)+p(\mathrm{~V})$ is substituted by $p(\mathrm{TV})$ on the right side of the expression. To perform this substitution, $p(T)$ has to be added to both sides of Eq. (3):

$p(\mathrm{TAV})+p(\mathrm{~T}) \leq p(\mathrm{TA})+p(\mathrm{~T})+p(\mathrm{~V})$

$p(\mathrm{TAV})+p(\mathrm{~T}) \leq p(\mathrm{TA})+p(\mathrm{TV})$

In a final step, $p(\mathrm{~A})+p(\mathrm{~V})$ is substituted by $p(\mathrm{AV})$. Again, $p(\mathrm{~A})$ and $p(\mathrm{~V})$ are first added to both sides of Eq. (4):

$p(\mathrm{TAV})+p(\mathrm{~T})+p(\mathrm{~A})+p(\mathrm{~V}) \leq p(\mathrm{TA})+p(\mathrm{TV})+p(\mathrm{~A})$ $+p(\mathrm{~V})$

$p(\mathrm{TAV})+p(\mathrm{~T})+p(\mathrm{~A})+p(\mathrm{~V}) \leq p(\mathrm{TA})+p(\mathrm{TV})$ $+p(\mathrm{AV})$

If Eq. (5) is violated, a specific trisensory interaction can be assumed. By contrast, if Eq. (5) holds, bisensory interactions between audition and vision, audition and touch, and vision and touch are sufficient to explain the redundancy gain in trimodal stimuli.

Since the left side of Eq. (5) approaches 4.0 at the higher percentile range, whereas the right side approaches 3.0, Eq. (5) can only be applied for the lower percentile range of the reaction time distribution. Significance of race model violations was tested for each $5 \%$ bin using a binomial test.

\section{Acknowledgment}

This study was supported by the Emmy Noether grant Ro 1226/ $4-1 / 2 / 3$ to $B R$ of the German Research Foundation (DFG).

\section{R E F E R E N C E S}

Barth, D.S., Goldberg, N., Brett, B., Di, S., 1995. The spatiotemporal organization of auditory, visual, and auditory-visual evoked potentials in rat cortex. Brain Res. 678, 177-190.

Busse, L., Woldorff, M.G., 2003. The ERP omitted stimulus response to "no-stim" events and its implications for fast-rate event-related fMRI designs. NeuroImage 18, 856-864.

Diederich, A., 1992. Probability inequalities for testing separate activation models of divided attention. Percept. Psychophys. 52, 714-716.

Fort, A., Delpuech, C., Pernier, J., Giard, M.-H., 2002. Early auditory-visual interactions in human cortex during nonredundant target identification. Cogn. Brain Res. 14, 20-30.

Foxe, J.J., Morocz, I.A., Murray, M.M., Higgins, B.A., Javitt, D.C., Schroeder, C.E., 2000. Multisensory auditory-somatosensory interactions in early cortical processing revealed by high-density electrical mapping. Cogn. Brain Res. 10, 77-83.

Giard, M.-H., Peronnet, F., 1999. Auditory-visual integration during multimodal object recognition in humans: a behavioral and electrophysiological study. J. Cogn. Neurosci. 11, 473-490.

Gobbelé, R., Schürmann, M., Forss, N., Juottonen, K., Buchner, H., Hari, R., 2003. Activation of the human posterior parietal and temporoparietal cortices during audiotactile interaction. NeuroImage 20, 503-511.

Gondan, M., Röder, B., in revision. Testing trisensory interactions.

Gondan, M., Niederhaus, B., Rösler, F., Röder, B., 2005. Multisensory processing in the redundant target effect: a behavioral and event-related potential study. Percept. Psychophys. 67, 713-726.

Miller, J., 1982. Divided attention: evidence for coactivation with redundant signals. Cognit. Psychol. 14, 247-279.

Molholm, S., Ritter, W., Murray, M.M., Javitt, D.C., Schroeder, C.E., Foxe, J.J., 2002. Multisensory auditory-visual interactions during early sensory processing in humans: a high-density electrical mapping study. Cogn. Brain Res. 14, 115-128.

Murray, M.M., Molholm, S., Michel, C.M., Heslenfeld, D.J., Ritter, W., Javitt, D.C., Schroeder, C.E., Foxe, J.J., 2005. Grabbing your ear: rapid auditory-somatosensory multisensory interactions in low-level sensory cortices are not constrained by stimulus alignment. Cereb. Cortex 5, 963-974.

Raab, D.H., 1962. Statistical facilitation of simple reaction times. Trans. N. Y. Acad. Sci. 24, 574-590.

Schlack, A., Strebing-D'Angelo, S., Hartung, K., Hoffmann, K.-P., Bremmer, F., 2005. Multisensory space representations in the Macaque ventral intraparietal area. J. Neurosci. 25, 4616-4625.

Schröger, E., Widmann, A., 1998. Speeded responses to audiovisual changes result from bimodal integration. Psychophysiology 35 , 755-759.

Schürmann, M., Kolev, V., Menzel, K., Yordanova, J., 2002. Spatial coincidence modulates interaction between visual and somatosensory evoked potentials. NeuroReport 13, 779-783.

Simson, R., Vaughan Jr., H.G., Ritter, W., 1976. The scalp topography of potentials associated with missing visual or auditory stimuli. Electroencephalogr. Clin. Neurophysiol. 40, 33-42.

Sternberg, S., 2001. Separate modifiability, mental modules, and the use of pure and composite measures to reveal them. Acta Psychol. 106, 147-246.

Teder-Sälejärvi, W.A., McDonald, J.J., Di Russo, F., Hillyard, S.A., 2002. An analysis of audio-visual crossmodal integration by means of event-related potential (ERP) recordings. Cogn. Brain Res. 14, 106-114.

Wallace, M.T., Stein, B.E., 2001. Sensory and multisensory responses in the newborn monkey superior colliculus. J. Neurosci. 21, 8886-8894.

Wallace, M.T., Meredith, M.A., Stein, B.E., 1992. Integration of multiple sensory modalities in cat cortex. Exp. Brain Res. 91, 484-488.

Walter, W.G., Cooper, R., Aldridge, V.J., McCallum, W.C., Winter, A.L., 1964. The contingent negative variation. Nature 203, 380-384.

Welch, R.B., Warren, D.H., 1986. Intersensory interactions. In: Boff, K.R., Kaufman, L., Thomas, J.P. (Eds.), Handbook of Perception and Human Performance. Wiley, New York, pp. 25/1-25/36. 